\title{
Large Capacity ATM Switching Fabric Using Three Dimensional Optical CDMA Technology
}

\author{
Che-Li Lin and Jingshown Wu \\ Room 519 \\ Department of Electrical Engineering \\ National Taiwan University \\ Taipei, Taiwan 106, R.O.C. \\ lincl@r528d.ee.ntu.edu.tw
}

\begin{abstract}
In this paper, a novel asynchronous transfer mode (ATM) switching fabric using three dimensional optical code division multiple access (CDMA) technology is presented. The proposed three dimensional codes, temporalspatial/wavelength (TSW) codes, have out-of-phase autocorrelation and cross correlation equal to zero and one, respectively. The spectral slicing technology is utilized to achieve wavelength encoding and decoding. The results show that the switching fabric has no internal blocking and the number of input/output ports can reach up to 1369. The cell loss without considering the output blocking is smaller than $10^{-8}$. The total throughput can be as large as $1.1 \mathrm{~Tb} / \mathrm{s}$.
\end{abstract}

\section{Introduction 1}

ATM switches are the core components of the ATM network, which has been emerging as one of the best candidates for integrated broadband communications. Because electronic ATM switches are limited by the bottleneck of the electronic processing speed, various photonic ATM switches have been proposed [1], [2]. Among these different designs, the optical time, space, or wavelength division multiple access techniques are most widely used. The photonic ATM switches based on optical CDMA are presented recently [3],[4]. These "broadcast-and-select" CDMA switches are asynchronous and efficient, especially for bursty traffic in local area networks.

For a CDMA based ATM switch to support a large number of simultaneous access ports, not only the size of the code set but also the autocorrelation and cross correlation properties are very important. If the optical orthogonal

\footnotetext{
${ }^{1}$ This work is supported by the National Science Council, R.O.C. under the grant NSC 88-2215-E-002-035.
}

codes (OOC's) are used as the spreading sequences, the most serious problem is that the number of simultaneous access ports is much smaller than that of the available codes [5]. Besides, because the data bits are spread only in the time domain, the spreading sequences are all very lengthy, which makes the chip rate much higher than the bit rate, thus increasing the difficulty of realization. Recently, two dimensional codes have been proposed to improve the system performance. For example, the design and experimental results of temporal/spatial (T/S) incoherent optical CDMA networks have been presented [6]-[8]. In addition to having shorter spreading sequences, these $T / S$ codes possess other advantages, such as smaller out-of-phase autocorrelation and cross correlation, and lower encoding/decoding loss. Another kind of two dimensional codes called multiwavelength optical orthogonal codes (MWOOC's) spreads the data bit in both the wavelength and time domains to support large numbers of subscribers and simultaneous users in fiber-optic CDMA networks [9].

In this paper, we present a new family of three dimensional codes, which is named the TSW codes because the data bits are spread in temporal, spatial, and wavelength domains. In addition to large code sets, the out-of-phase autocorrelation and cross correlation of the proposed codes are equal to zero and one, respectively. Using the TSW codes, we propose a photonic ATM switching fabric in which the spectral slicing technology and the arrayed waveguide grating (AWG) are utilized to achieve wavelength encoding and decoding [10]. Because there are no optical splitters or combiners in the proposed architecture, the corresponding splitting or combining loss can be eliminated compared to one or two dimensional codes. Compared with OOC's and T/S AML codes, the TSW codes can afford shorter temporal length under the same number of simultaneous access ports and bit error probability [8]. Because the TSW codes split the system complexities evenly in three dimensions, the bot- 
tleneck of very high chip rate can be relieved, thereby making the system more viable. When the system parameters are properly set, the number of simultaneous access ports of the switching fabric can be the same as the number of available codes with cell loss probability smaller than $10^{-8}$.

The remainder of this paper is organized as follows. In Section II, the TSW codes are presented. The ATM switching fabric is described in Section III. The system performance analysis is given in Section IV. Section V presents the numerical results and comparisons with other systems. Some discussions are also given in this section. Finally, Section VI concludes this paper.

\section{Design of TSW codes}

The TSW codes can be characterized by $N_{A}\left(L, R, C, p, W, \lambda_{a}, \lambda_{c}\right)$, where $N_{A}$ is the number of available codes, $L$ is the temporal length, $R$ is the number of spatial channels, $C$ is the number of wavelength channels, $p$ is the number of pulses per spatial channel, $W$ is the weight of the codes, $\lambda_{a}$ is the out-of-phase autocorrelation peak, and $\lambda_{c}$ is the cross-correlation peak. In our switching fabric, which will be shown in the following section, $C$ is equal to $R$. To restrict $\lambda_{c}=1, p$ must be 1 . Therefore, we have $W=C=R$.

A TSW code can be represented by an $R \times L$ matrix. The $y$ th code in the $x$ th group can be expressed by the matrix $A^{x, y}$, where

$A^{x, y}=\left[a_{i, j}^{x, y}\right]_{R \times L}=\left[\begin{array}{cccc}\text { Time } & & \rightarrow \\ a_{1,1}^{x, y} & a_{1,2}^{x, y} & \cdots & a_{1, y}^{x, y} \\ a_{2,1}^{x, y} & a_{2,2}^{x, y} & \cdots & a_{2, L}^{x, y} \\ \vdots & \vdots & & \vdots \\ \vdots & \vdots & & \vdots \\ a_{R, 1}^{x, y} & a_{R, 2}^{x, y} & \cdots & a_{R, L}^{x, y}\end{array}\right] \begin{aligned} & S \\ & p \\ & e \\ & e\end{aligned}$

and

$$
a_{i, j}^{x, y}=\left\{\begin{array}{c}
{[(i+y-2) \bmod R]+1} \\
\text { if } j=[(x-1)(i-1) \bmod L]+1 \\
0, \quad \text { otherwise }
\end{array}\right.
$$

where $x, j \in\{1,2, \cdots, L\}$ and $y, i \in\{1,2, \cdots, R\}$. If $a_{i, j}^{x, y}=0$, there is no optical pulse transmitted in the $j$ th time slot of the $i$ th spatial channel. If $a_{i, j}^{x, y}=n, n \in$ $\{1,2, \cdots, R\}$, there is an optical pulse of the $n$th wavelength channel transmitted in the $j$ th time slot of the $i$ th spatial channel. For example, if $R=3$, the codes have three spatial and wavelength channels. When $L=5$, the temporal length of the code is five. According to (1) and (2), the code $A^{4,2}$ can be expressed by the following $3 \times 5$

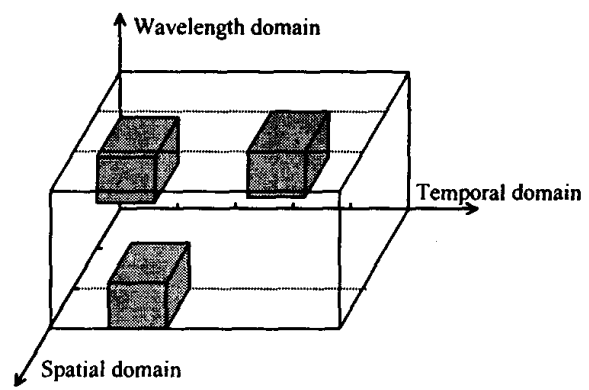

Figure 1. The three dimensional representation of the code $A^{4,2}$.

matrix.

$$
A^{4,2}=\left[\begin{array}{lllll}
2 & 0 & 0 & 0 & 0 \\
0 & 0 & 0 & 3 & 0 \\
0 & 1 & 0 & 0 & 0
\end{array}\right]
$$

The three dimensional representation of the code $A^{4,2}$ is also shown in Fig. 1, where pulses are at $(1,1,2),(2,4,3)$, and $(3,2,1)$ in the coordinates (Space, Time, Wavelength).

The autocorrelation for a TSW code $A^{x, y}$ is defined as follows.

$$
C_{x, y}(l)=\sum_{i=1}^{R} \sum_{j=1}^{L} \delta\left(a_{i, j}^{x, y}, a_{i,(j+l) \bmod L}^{x, y}\right) \cdot u\left(a_{i, j}^{x, y}\right)
$$

where

$$
\delta(m, n)= \begin{cases}1, & \text { if } m=n \\ 0, & \text { otherwise }\end{cases}
$$

and

$$
u(m)= \begin{cases}1, & \text { if } m \geq 1 \\ 0, & \text { otherwise }\end{cases}
$$

$C_{x, y}(l)$ satisfies

$$
C_{x, y}(l)= \begin{cases}W, & \text { if } l \bmod L=0 \\ \leq \lambda_{a}, & \text { otherwise }\end{cases}
$$

The cross correlation for two TSW codes $A^{x, y}$ and $A^{x^{\prime}, y^{\prime}}$ is defined as follows.

$$
C_{x, y, x^{\prime}, y^{\prime}}(l)=\sum_{i=1}^{R} \sum_{j=1}^{L} \delta\left(a_{i, j}^{x, y}, a_{i,(j+l) \bmod L}^{x^{\prime}, y^{\prime}}\right) \cdot u\left(a_{i, j}^{x, y}\right)
$$

And $C_{x, y, x^{\prime}, y^{\prime}}(l)$ satisfies

$$
C_{x, y, x^{\prime}, y^{\prime}}(l) \leq \lambda_{c}, \quad \text { if } 0 \leq l \leq L-1
$$

To have the maximal number of codes under the condition that $\lambda_{a}=0$ and $\lambda_{c}=1, L$ must be a prime number, and 
$R \leq L$ [8]. In this paper, we consider only the codes which satisfy the above conditions. From (1) and (2), the number of available codes, $N_{A}$, satisfies the following equation.

$$
N_{A}=R \cdot L \text {. }
$$

\section{ATM switching fabric}

The proposed ATM switching fabric as shown in Fig. 2 consists input port modules, $N_{A} \times L$ couplers, and output port modules. Each input port module is composed of a broadband light source, an external modulator [11], an $1 \times R$ space switch, an $R \times R$ AWG, and a set of $R$ tunable optical delay lines. The broadband light source, which may be shared among more than one modules, can be a superluminescent diode, a spectrally uniform broadband amplified spontaneous emission (ASE) source, or various erbiumdoped fiber amplifier ASE based sources [10]. No optical pulses are sent if the transmitted bit is 0 . Therefore, the external modulator turns on for one chip time, $T_{c}$, only when the transmitted bit is 1 . Here one chip time is equivalent to one time slot in the above section. The $1 \times R$ space switch is used to switch the broadband optical pulse to one of the $R$ input ports of the $R \times R$ AWG. The wavelength channel which routes from the $i$ th input port to the $j$ th output port of the AWG is $[(i+j-2) \bmod R]+1$. For example, the wavelength assignment table of the $4 \times 4$ AWG is shown in Fig. 3 . If the optical pulses made up of $\lambda_{1}, \lambda_{2}, \lambda_{3}$, and $\lambda_{4}$ are input at the second port of the $4 \times 4$ AWG, they will get out from the fourth, first, second, and third output ports, respectively. Finally, the $R$ tunable optical delay lines introduce unequal delays to the output pulses from the AWG. The input port modules are connected by $R N_{A} \times L$ star couplers as shown in Fig. 2. Because it is a tunable-transmitter/fixed-receiver system, the optical delay lines and the $R \times R$ AWG can be shared among $R$ different receivers. The optical delay lines are fixed. The $R$ output ports of the AWG are connected to optical hardlimiters. The relation between the input optical power, $z$, and the output optical power of the optical hardlimiter, $g(z)$, is defined as follows.

$$
g(z)= \begin{cases}R P_{u}, & z \geq R P_{u} \\ 0, & 0 \leq z<R P_{u}\end{cases}
$$

where $P_{u}$ is the unit optical power. Then the avalanche photodiode (APD) converts the optical power into electrical current and the followed OOK decoder makes the decision over the last chip time of the code to obtain the desired signal.

In the proposed ATM switching fabric, the only element operating at the chip rate is the external modulator. A millimeter-wave $\mathrm{Ti}: \mathrm{LiNbO}_{3}$ optical modulator operating at $40 \mathrm{~Gb} / \mathrm{s}$ has been proposed [11]. The $1 \times R$ space switches and the tunable optical delay lines need not to be tuned until an ATM cell is completely transmitted.

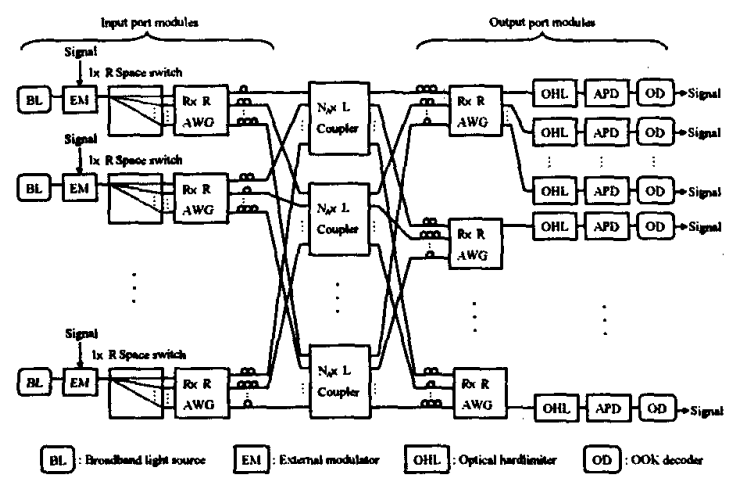

Figure 2. The block diagrams of the TSW codes encoders, decoders, and the fiber-

\begin{tabular}{|c|c|c|c|c|}
\hline & \multicolumn{4}{|c|}{ Output ports } \\
\hline & 1 & 2 & 3 & 4 \\
\hline \multirow{4}{*}{ 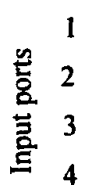 } & $\lambda_{1}$ & $\lambda_{2}$ & $\lambda_{3}$ & $\lambda_{4}$ \\
\hline & $\lambda_{2}$ & $\lambda_{3}$ & $\lambda_{4}$ & $\overline{\lambda_{1}}$ \\
\hline & $\lambda_{3}$ & $\lambda_{4}$ & $\lambda_{1}$ & $\lambda_{2}$ \\
\hline & $\lambda_{4}$ & $\lambda_{1}$ & $\lambda_{2}$ & $\lambda_{3}$ \\
\hline
\end{tabular}
optic CDMA network fabric.

Figure 3. The wavelength assignment table of the $4 \times 4$ AWG.

\section{System Performances Analysis}

In this section, the bit error probability (BEP) of the proposed switching fabric is analyzed. It is assumed that each port is assigned a unique TSW code. Then the BEP conditional on $N$ simultaneous access ports can be written as

$$
\begin{aligned}
& P_{\mathrm{e}}=\operatorname{Pr}\{\text { bit error } \mid N=n\}=\operatorname{Pr}\left\{I_{p}>\theta \mid N=n, b=0\right\} \\
& \cdot \operatorname{Pr}\{b=0\}+\operatorname{Pr}\left\{I_{p}<\theta \mid N=n, b=1\right\} \cdot \operatorname{Pr}\{b=1\}
\end{aligned}
$$

where $I_{p}$ is the average output current of the APD over the last chip time of the code, $\theta$ is the threshold level of the OOK decoder, and $b$ is the desired received bit. The conditional distribution function of $I_{p}$ when $N=n$ and $b=0$ can be written as

$\operatorname{Pr}\left\{I_{p}=i_{p} \mid N=n, b=0\right\}=\frac{1}{\sqrt{2 \pi \sigma_{i_{p}}^{2}, 0}} e^{-\left(i_{p}-\mu_{i_{p}, 0}\right)^{2} / 2 \sigma_{i_{p}, 0}^{2}}$

where the mean $\mu_{i_{p}, 0}$ can be expressed as

$$
\mu_{i_{p}, 0}=M R_{A} P_{i n, 0}
$$




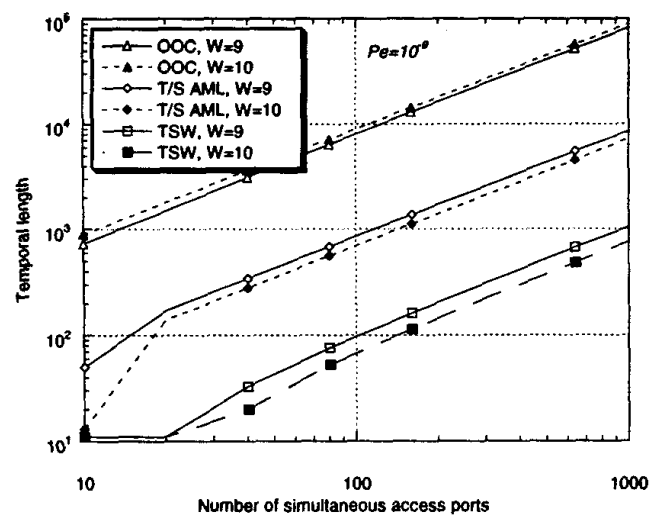

Figure 4. Temporal length comparisons among three types of fiber-optic CDMA codes.

$M$ is the average APD gain, $R_{A}$ is the responsivity of the APD, and $P_{i n, 0}$ is the average received optical power over the last chip time of the code when $b=0$. And

$\sigma_{i_{p}, 0}^{2}=4\left(k_{B} T / R_{L}\right) F_{n} \Delta f+2 q M^{2} F_{A}\left(R_{A} P_{i n, 0}+I_{d}\right) \Delta f$

where $k_{B}$ is Boltzmann's constant, $T$ is the absolute temperature, $R_{L}$ is the load resistance, $F_{n}$ is the noise figure of the preamplifier, $\Delta f$ is the effective noise bandwidth, $q$ is an electron charge, $F_{A}$ is the excess noise factor of the APD and is given by

$$
F_{A}=k_{A} M+\left(1-k_{A}\right)(2-1 / M)
$$

where $k_{A}$ is the APD effective ionization ratio, and $I_{d}$ is the dark current.

Because there is an optical hardlimiter before the APD, $P_{i n, 0}$ has only two possible values, 0 and $R P_{u}$. The probability of $P_{i n, 0}$ equal to zero is given by

$$
\begin{gathered}
\operatorname{Pr}\left\{P_{i n, 0}=0\right\}=\sum_{m=0}^{\min \{R-1, N-1\}} \sum_{i=\max \{m, L+N-L R-1\}}^{\min \{N-1, L-1\}} \\
\frac{\left(\begin{array}{c}
L-1 \\
i
\end{array}\right)\left(\begin{array}{c}
L R-L \\
N-i-1
\end{array}\right)}{\left(\begin{array}{c}
L R-1 \\
N-1
\end{array}\right)}\left(\begin{array}{c}
i \\
m
\end{array}\right)\left(\frac{R}{2 L}\right)^{m}\left(1-\frac{R}{2 L}\right)^{i-m}
\end{gathered}
$$

where the function $\max \{\alpha, \beta\}$ and $\min \{\alpha, \beta\}$ are the $\max$ imum and minimum of $\alpha$ and $\beta$, respectively. And

$$
\operatorname{Pr}\left\{P_{i n, 0}=R P_{u}\right\}=1-\operatorname{Pr}\left\{P_{i n, 0}=0\right\}
$$

The conditional distribution function of $I_{p}$ when $N=n$

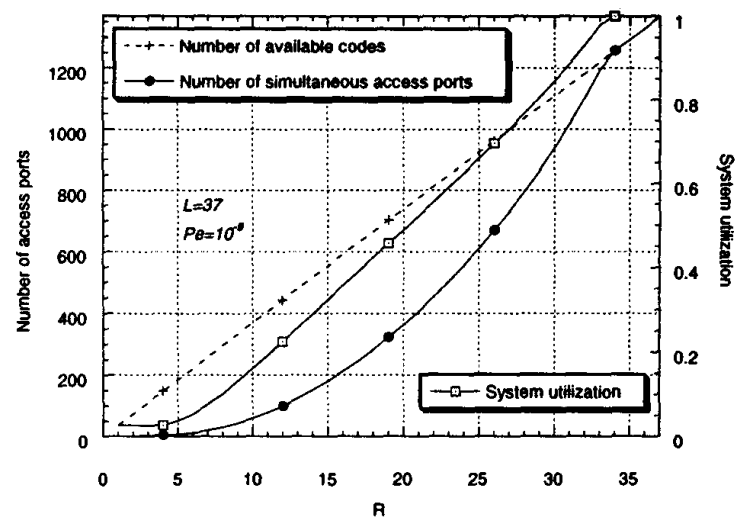

Figure 5. The number of access ports and system utilization versus $R$.

and $b=1$ is expressed as

$\operatorname{Pr}\left\{I_{p}=i_{p} \mid N=n, b=1\right\}=\frac{1}{\sqrt{2 \pi \sigma_{i_{p}, 1}^{2}}} e^{-\left(i_{p}-\mu_{i_{p}, 1}\right)^{2} / 2 \sigma_{i_{p}, 1}^{2}}$

where the mean $\mu_{i_{p}, 1}$ and the variance $\sigma_{i_{p}, 1}^{2}$ are the same as the expressions in (14) and (15) except that $P_{i n, 0}$ is changed to $P_{i n, 1} . P_{i n, 1}$, whose value is $R P_{u}$, is the average received optical power over the last chip time of the code when $b=1$. The threshold level, $\theta$, is set to a suboptimum value as [12]

$$
\theta=\left.\frac{\mu_{i_{p}, 0} \cdot \sigma_{i_{p}, 1}+\mu_{i_{p}, 1} \cdot \sigma_{i_{p}, 0}}{\sigma_{i_{p}, 1}+\sigma_{i_{p}, 0}}\right|_{P_{i n, 1}=R P_{u}, P_{i n, 0}=0}
$$

\section{Numerical results and discussions}

In this example, some system parameters are given as follows: $R_{L}=1000 \Omega, F_{n}=3 \mathrm{~dB}, T=300 \mathrm{~K}, \Delta f=$ $10 \mathrm{GHz}, M=100, R_{A}=0.8 \mathrm{~A} / \mathrm{W}, k_{A}=0.02, R \cdot P_{u}=$ $8 \times 10^{-7} \mathrm{~W}$. Fig. 4 shows the temporal lengths of the proposed TSW code, OOC's, and T/S AML codes, where $W$ 's are the weights of the corresponding codes [5],[8]. Given the same number of simultaneous access ports and bit error probability, the temporal length of the TSW codes is much shorter than those of T/S AML codes and the OOC's. The trade-off is quite clear: the more dimensions we use, the shorter temporal length of the code is. Using the TSW codes, however, splits the system complexities evenly in three dimensions, so the system constraints can be relaxed. In Fig. 5, under the condition that the value of $L$ is fixed at 37 , the number of available codes, $N_{A}$, increases linearly with $R$. The number of simultaneous access ports grows 


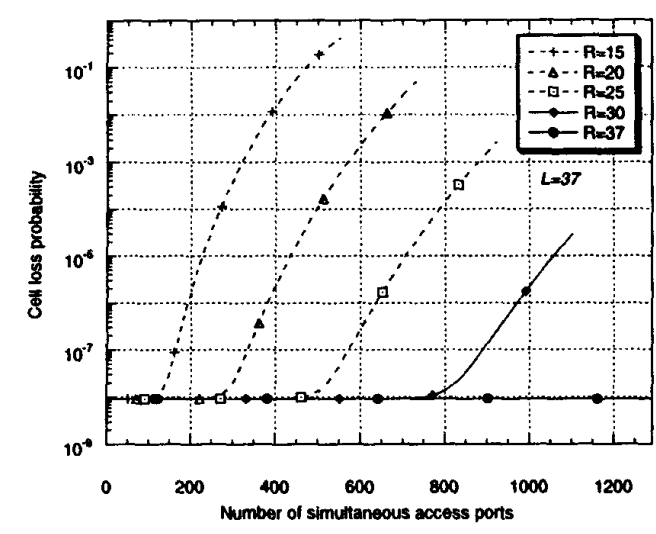

Figure 6. The relation between cell loss probability and the number of access ports.

faster when $R$ gets larger. Another figure of merit, system utilization, is defined as follows.

$$
U_{s}=\left.\frac{N}{N_{A}}\right|_{P_{e}=10^{-9}}
$$

It is seen that the system utilization increases with $R$. When $R \geq 34, U_{s}$ equals to unity, which is because that the maximum number of interference users, $L-1$, and the weight of code, $R$, are almost equal. Therefore, we come to the conclusion that the value of $R$ should be as close to $L$ as possible to have the best performance.

The cell loss probability, $P_{c l}$, is defined as $P_{c l}=1-$ $\left(1-P_{e}\right)^{B_{c}}$, where $B_{c}$ is the number of bits per cell and is 424 for an ATM cell. The relation between $P_{c l}$ and $N$ for various $R$ is shown in Fig. 6. The larger $R$ is, the better the performance is. When $R=L=37, P_{c l}$ is smaller than $10^{-8}$ with $N=1369$. Fig. 7 shows the switching speed per port, $R_{b}$, and total throughput, $T_{h}$. Because the temporal length of the code is $L$, the chip rate, $R_{c}$, is defined as $L R_{b}$. With constant $R_{c}$, we see that $T_{h}$ grows with $L$ while $R_{b}$ decreases with $L$. The power budget of the proposed architecture is also investigated. The various link parameters are given in Table 1 . The system margin, $M_{S}$, is calculated as follows.

$$
\begin{aligned}
M_{S}= & P_{B L}-L_{E M}-L_{S S}-L_{T D} \\
& -2 L_{A W G}-L_{C}-L_{F D}-R \cdot P_{u}
\end{aligned}
$$

If we have $R \cdot P_{u}=8 \times 10^{-7} \mathrm{~W}$, and $N_{A}=1369$, the value of $M_{S}$ is $5.6 \mathrm{~dB}$.

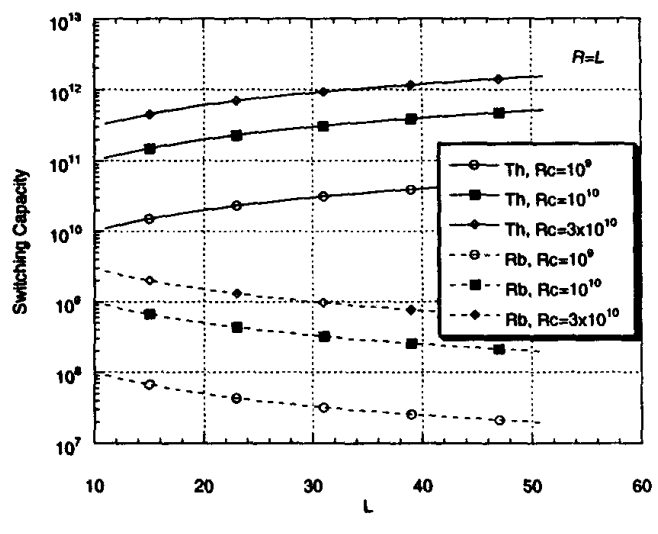

Figure 7. The relations among the total throughput, switching speed per port, and $L$.

\section{Conclusion}

In this paper, the construction of a new family of three dimensional codes, TSW codes, and the corresponding ATM switching fabric are presented. The TSW codes have the autocorrelation sidelobe and cross correlation peaks equal to zero and one, respectively. We utilize the spectrum slicing technology and the AWG in the system, which has the advantages, such as no combining or splitting loss and the relatively small temperature dependence of the passband position. Based on the analysis and numerical results, we know that the TSW codes have superior performance compared with one or two dimensional codes. If the system parameters are set properly, the number of simultaneous access ports can be the same as the number of available codes with cell loss probability smaller than $10^{-8}$ and total throughput as large as $1.1 \mathrm{~Tb} / \mathrm{s}$.

\section{References}

[1] Y. Nakahira, H. Sunahara, and Y. Oie, "Comparative evaluation of photonic ATM switch architectures," IEICE Trans. Commun., vol. E81-B, no. 2, pp. 473-481, Feb, 1998

[2] A. Misawa and M. Tsukada, "Broadcast-and-select photonic ATM switch with frequency division multiplexed output buffers," J. Lightwave Technol., vol. LT-15, no. 10, pp. 1769-1777, Oct 1997

[3] K. Kamakura, T. Ohtsuki, and I. Sasase, "An ATMbased optical code division multiplexing transport network using coherent ultrashort pulses," in Proc. 


\begin{tabular}{lll}
\hline Name & Symbol & Value \\
\hline $\begin{array}{l}\text { Broadband light source } \\
\text { power }\end{array}$ & $P_{B L}$ & $100 \mathrm{~mW}$ \\
$\begin{array}{l}\text { Insertion loss of external } \\
\text { modulator }\end{array}$ & $L_{E V}$ & $2 \mathrm{~dB}$ \\
\hline $\begin{array}{l}\text { Insertion loss of the } 1 \times R \\
\text { space switch }\end{array}$ & $L_{S S}$ & $2 \mathrm{~dB}$ \\
\hline $\begin{array}{l}\text { Insertion loss of the RxR } \\
\text { AWG }\end{array}$ & $L_{A H G}$ & $3 \mathrm{~dB}$ \\
\hline $\begin{array}{l}\text { Insertion loss of the } \\
\text { tunable delay line }\end{array}$ & $L_{T D}$ & $3 \mathrm{~dB}$ \\
\hline $\begin{array}{l}\text { Insertion loss of the } N_{A} x L \\
\text { coupler }\end{array}$ & $L_{C}$ & $10 \log N_{A} \mathrm{~dB}$ \\
\hline $\begin{array}{l}\text { Insertion loss of the fixed } \\
\text { delay line }\end{array}$ & $L_{F D}$ & $1 \mathrm{~dB}$ \\
\hline System margin & $M_{S}$ & \\
\hline
\end{tabular}

Table 1. LINK PARAMETERS

GLOBECOM'98, Sydney, Australia, Nov. 1998, pp. 3215-3220

[4] J.-G. Zhang, A. B. Sharma, and W. C. Kwong, "Optical CDMA-based ATM switches supporting equal-bitrate and variable-bit-rate communication services," in Proc. GLOBECOM'98, Sydney, Australia, Nov. 1998, pp. 3221-3226

[5] F. R. K. Chung, J. A. Salehi, and V. K. Wei, "Optical orthogonal codes: design, analysis, and applications," IEEE Trans. Inform. Theory, vol. 35, no. 3, pp. 595604, May 1989

[6] E. Park, A. J. Mendez, and E. M. Garmire, "Temporal/spatial optical CDMA networks-design, demonstration, and comparison with temporal networks," IEEE Photon. Technol. Lett., vol. 4, no. 10, pp. 11601162, Oct. 1992

[7] A. J. Mendez, J. L. Lambert, J.-M. Morookian, and R. M. Gagliardi, "Synthesis and demonstration of high speed, bandwidth efficient optical code division multiple access tested at $1 \mathrm{~Gb} / \mathrm{s}$ throughput," IEEE Photon. Technol. Lett., vol. 6, no. 9, pp. 1146-1149, Sep. 1994

[8] E. S. Shivaleela, K. N. Sivarajan, and A. Selvarajan, "Design of a new family of two-dimensional codes for fiber-optic CDMA networks," J. Lightwave Technol., vol. LT-16, no. 4, pp. 501-508, Apr. 1998

[9] G.-C. Yang, and W. C. Kwong, "Performance comparison of multiwavelength CDMA and WDMA + CDMA for fiber-optic networks," IEEE Trans. Commun., vol. 45, no. 11, pp. 1426-1434, Nov. 1997
[10] R. D. Feldman, "Crosstalk and loss in wavelength division multiplexed systems employing spectral slicing," J. Lightwave Technol., vol. LT-15, no. 11, pp. 1823-1831, Nov. 1997

[11] K. Noguchi, O. Mitomi, and H. Miyazawa, "Millimeter-wave $\mathrm{Ti}$ : $\mathrm{LiNbO}_{3}$ optical modulators," J. Lightwave Technol., vol. LT-16, no. 4, pp. 615-619, Nov. 1998

[12] M. M. Liu, Principles and applications of optical communications. Boston: IRWIN, 1996. 